\title{
Short-Term Evaluation of Dupilumab Effects in Patients with Severe Asthma and Nasal Polyposis
}

\author{
Corrado Pelaia (D) \\ Nicola Lombardo (iD ${ }^{2}$ \\ Maria Teresa Busceti ${ }^{1}$ \\ Giovanna Piazzetta ${ }^{2}$ \\ Claudia Crimi $\mathbb{D D}^{3}$ \\ Cecilia Calabrese (D) ${ }^{4}$ \\ Alessandro Vatrella $\mathbb{( D}^{5}$ \\ Girolamo Pelaia (D) ${ }^{\prime}$ \\ 'Department of Health Sciences, Magna \\ Graecia University of Catanzaro, \\ Catanzaro, Italy; ${ }^{2}$ Department of Medical \\ and Surgical Sciences, Magna Graecia \\ University of Catanzaro, Catanzaro, Italy; \\ ${ }^{3}$ Department of Clinical and \\ Experimental Medicine, University of \\ Catania, Catania, Italy; ${ }^{4}$ Department of \\ Translational Medical Sciences, Luigi \\ Vanvitelli University of Campania, Naples, \\ Italy; ${ }^{5}$ Department of Medicine, Surgery \\ and Dentistry, University of Salerno, \\ Salerno, Italy
}

Correspondence: Corrado Pelaia Department of Health Sciences, Magna Graecia University of Catanzaro, Catanzaro, Italy

Tel + $390961364-7007$

Email pelaia.corrado@gmail.com
Background: Having been approved for biological treatment of atopic dermatitis, dupilumab has also been recently licensed as add-on therapy for severe asthma and nasal polyposis. With regard to the latter diseases, few real-life clinical investigations have been carried out to date.

Objective: The primary end point of this single-center observational study was to evaluate in a real-life setting the short-term therapeutic effects of dupilumab in patients with severe asthma and nasal polyposis.

Methods: At baseline and after 4 weeks of add-on therapy with dupilumab, several clinical and functional parameters were assessed in 20 patients with severe asthma and nasal polyposis, including both allergic and nonallergic subjects.

Results: After 4 weeks of treatment with dupilumab, all patients experienced remarkable improvement in both severe asthma and nasal polyposis. In particular, asthma-control test and sinonasal outcome test 22 scores had significantly increased $(p<0.0001)$ and decreased $(p<0.0001)$, respectively. Oral corticosteroid intake got to zero within 4 weeks $(p<0.0001)$. Moreover, in week 4, significant increases were detected with regard to both prebronchodilator forced expiratory volume in the first second $(p<0.01)$ and forced vital capacity (FVC; $p<0.05$ ). At the same time point, dupilumab had significantly reduced residual volume $(p<0.0001)$ and total lung capacity $(p<0.001)$, whereas it had enhanced forced midexpiratory flow of $25 \%-75 \%$ FVC $(p<0.01)$ and peak expiratory flow $(p<0.01)$. After 4 weeks of treatment, dupilumab had also lowered levels of fractional exhaled nitric oxide $(p<0.0001)$. Conclusion: The results of this real-life study suggest that dupilumab can be utilized in both allergic and nonallergic patients with severe asthma and nasal polyposis as a valuable add-on biological therapy with rapid onset of action.

Keywords: severe asthma, nasal polyposis, interleukin 4 receptor, dupilumab

\section{Introduction}

Severe asthma is characterized by heterogeneous phenotypes underpinned by several inflammatory patterns, which are driven by complex pathobiological mechanisms known as endotypes. ${ }^{1-3}$ Among the latter, two distinct entities have been identified and defined as type 2 (T2-high) or non-type 2 (T2-low) asthma, respectively. $^{4-9}$ The most important cellular elements involved in the development, persistence, and amplification of type 2 asthma are T-helper $2\left(\mathrm{~T}_{\mathrm{h}} 2\right)$ lymphocytes and group 2 innate lymphoid cells (ILC2). ${ }^{10-13}$ Upon activation, $\mathrm{T}_{\mathrm{h}} 2$ and ILC2 cells release large amounts of IL4, IL13, and IL5. ${ }^{5,14}$ IL4 induces immunoglobulin class switching, thereby stimulating B lymphocytes to synthesize IgE. ${ }^{15}$ IL13 cooperates with IL4 in promoting IgE production, and is also responsible for bronchial 
hyperresponsiveness, goblet-cell metaplasia, and proliferation of airway fibroblasts and smooth-muscle cells. ${ }^{16,17}$ IL13 also upregulates the expression of the inducible isoform of nitric oxide synthase (iNOS), thus increasing the emission of NO from airway epithelial cells, as well as the levels of fractional NO in exhaled breath (FeNO). ${ }^{18}$ IL5 plays a pivotal pathogenic role in eosinophilic inflammation by inducing the differentiation, survival, and activation of eosinophils. ${ }^{19}$

Therefore, high serum concentrations of IgE, minimum FeNO levels of 20-25 parts per billion (ppb), and blood eosinophils counts of at least $150-300$ cells/ $\mu \mathrm{L}$ are considered reliable biomarkers of T2-high asthma. ${ }^{2}$ Given the key biological links connecting these biomarkers with the aforementioned cytokines, it can be reasonably inferred that IL4, IL13, IL5, and their receptors represent suitable molecular targets for biological therapies for severe type 2 asthma, refractory to both standard inhaled treatments and oral corticosteroids (OCS). ${ }^{20-22}$ Within such a therapeutic context, very interesting pharmacologic features characterize dupilumab, a fully human $\mathrm{IgG}_{4}$ monoclonal antibody that binds specifically to the $\alpha$ subunit of the IL4 receptor (IL4R $\alpha){ }^{23}$ Because utilization of IL4R $\alpha$ is shared by IL4 and IL13, which activate the type I (IL4R $\alpha / \gamma \mathrm{c}$ ) and type II (IL4R $\alpha / \operatorname{IL} 13 R \alpha 1$ ) receptor dimers, respectively, dupilumab behaves as a receptor antagonist of both these cytokines. $^{24}$ Dupilumab has been recently evaluated by two phase III randomized clinical trials (RCTs; Liberty Asthma QUEST and Liberty Asthma VENTURE), ${ }^{25,26}$ the results of which have led to the approval of this biologic for add-on treatment of type 2 severe asthma. Dupilumab has also been licensed for biological therapy of severe chronic rhinosinusitis with nasal polyps (CRSwNP), effectively treatable with this drug, as shown by two phase III RCTs: Liberty NP SINUS-24 and Liberty NP SINUS-52. ${ }^{27-29}$ However, because of the recent introduction of dupilumab in clinical practice, few real-life studies have been published to date. ${ }^{30-32}$

Based on a real-world approach, we decided to investigate the short-term effects of dupilumab in patients with severe type 2 asthma and nasal polyposis. It has been shown that within 2 hours after the first administration, dupilumab effectively neutralized the functions of IL4R $\alpha$ expressed by $\mathrm{CD}^{+} \mathrm{T}$ cells and $\mathrm{CD} 19^{+} \mathrm{B}$ cells, and this receptor blockade persisted stably during the following 4 weeks. ${ }^{33}$ Therefore, the aim of our real-life, single-center study was to verify the rapidity of dupilumab therapeutic effects by evaluating at baseline and after 4 weeks of treatment several outcomes, including symptom control of severe asthma and CRSwNP, lung function, FeNO, and OCS consumption.

\section{Methods \\ Study Design and End Points}

We enrolled adult outpatients with severe eosinophilic asthma and nasal polyposis under treatment with dupilumab. Twenty subjects were recruited, eleven women and nine men, with a mean age of $55.8 \pm 15.17$ years and mean BMI of $26.55 \pm 5.68 \mathrm{~kg} / \mathrm{m}^{2}$. Mean baseline $\mathrm{FEV}_{1}$ was $67.8 \% \pm 20.01 \%$ of predicted value. Fourteen participants had positive skin-prick tests for perennial and/or seasonal aeroallergens. All 20 enrolled patients were referred to the Respiratory Unit and the Otolaryngology Unit of Magna Graecia University Hospital in Catanzaro, Italy. They complained of persistent asthma symptoms and CRSwNP, and needed high dosages of inhaled CS (ICS)long-acting $\beta_{2}$-adrenergic agonist combinations, along with long-acting muscarinic antagonists (LAMAs). Fourteen patients had not been previously treated with biologics, and the other six underwent a washout period of at least 2 months before switching to dupilumab from previous ineffective biological therapies with omalizumab (three patients), mepolizumab (two patients), or benralizumab (one patient). These patients were consecutively recruited at our University Clinical Center, and the only inclusion criteria regarded fulfilment of the items regulating eligibility for dupilumab treatment. CRSwNP was characterized by an eosinophilic pattern in all 20 patients. Skin-prick testing was performed by placing a drop of each allergen solution on the forearm marked by a skin marker pen, and every drop was pricked by a sterile lancet. Skin sensitivity was then determined by comparing the eventual reactive wheal with that produced by histamine. ${ }^{34}$ Blood eosinophil and basophil counts were obtained using automated hematology analyzers. ${ }^{35,36}$

At baseline, all participants had blood eosinophil counts of at least 150 cells/ $\mu \mathrm{L}$ and/or FeNO levels $>25$ $\mathrm{ppb}$, and/or required permanent or nearly continuous OCS treatment. All patients met the European Respiratory Society (ERS)/American Thoracic Society (ATS) criteria that define severe uncontrolled asthma. ${ }^{37}$ Study participants also reported a history of nasal polyposis, and a diagnosis of CRSwNP was obtained on the basis of clinical symptoms, nasal endoscopy, and computed tomography scans. ${ }^{38,39}$ Dupilumab was prescribed according to the 
existing eligibility indications and administered subcutaneously at an initial dose of $600 \mathrm{mg}$ (two injections of 300 $\mathrm{mg}$ at different sites), followed by maintenance dosing of $300 \mathrm{mg}$ every 2 weeks. $^{40}$ Baseline characteristics of patients are summarized in Table 1.

The main aim of this real-life investigation was to evaluate the effects of dupilumab on clinical, functional, and laboratory parameters. Asthma control test (ACT) scores, Sino-Nasal Outcome Test (SNOT-22) questionnaire scores, prednisone intake, forced expiratory volume in 1 second $\left(\mathrm{FEV}_{1}\right)$, forced vital capacity $(\mathrm{FVC})$, residual volume (RV), total lung capacity (TLC), forced midexpiratory flow of $25 \%-75 \%$ FVC $\left(\mathrm{FEF}_{25-75}\right)$, peak expiratory flow (PEF), FeNO levels, and blood eosinophil and basophil counts were assessed at baseline and 4 weeks after the first injection of dupilumab. Spirometry and body plethysmography were performed using the MasterScreen pulmonary function testing system and MasterScreen Body (Jaeger-Viasys; CareFusion, Höchberg, Germany), according to ATS/ERS guidelines. ${ }^{41}$ FeNO levels were measured with a Vivatmo Pro FeNO device (Bosch, Waiblingen, Germany) in accordance with ATS/ERS recommendations. ${ }^{42,43}$ For 2 hours after dupilumab administration, patients were monitored to detect the eventual onset of adverse reactions. Moreover, the possible occurrence of side effects was investigated through a once-weekly telephone call, as well as each time every patient underwent dupilumab injection.

This study met the standards of Good Clinical Practice and the principles of the Declaration of Helsinki. All patients signed a written informed consent. The study was also carried out in agreement with a statement from the local Ethical Committee of Calabria Region (Catanzaro, Italy; document 182-20th May 2021).

Table I Baseline patient features

\begin{tabular}{|l|l|}
\hline Age, mean $\pm \mathrm{SD}$, years & $55.80 \pm 15.17$ \\
Age of asthma onset, mean $\pm \mathrm{SD}$, years & $31.10 \pm 15.25$ \\
Duration of asthma, mean $\pm \mathrm{SD}$, years & $24.80 \pm 13.60$ \\
Female sex, $\mathrm{n}(\%)$ & $\mathrm{II}(55)$ \\
Weight, mean $\pm \mathrm{SD}, \mathrm{kg}$ & $70.20 \pm 14.64$ \\
Height, mean $\pm \mathrm{SD}, \mathrm{cm}$ & $164.6 \pm 10.57$ \\
$\mathrm{BMI}$, mean $\pm \mathrm{SD}, \mathrm{kg} / \mathrm{m}^{2}$ & $26.55 \pm 5.68$ \\
$\mathrm{FEV}$, mean $\pm \mathrm{SD}, \%$ predicted & $67.80 \pm 20.0 \mathrm{I}$ \\
$\mathrm{FEV} / \mathrm{FVC}$, mean $\pm \mathrm{SD}, \%$ & $69.45 \pm 8.92$ \\
Atopic subjects, $\mathrm{n}(\%)$ & $14(70)$ \\
Total serum IgE, median (IQR), IU/mL & $473.7 \pm 669.5$ \\
\hline
\end{tabular}

Abbreviations: $\mathrm{BMI}$, body-mass index; $\mathrm{FEV}_{1}$, forced expiratory volume in I second; FVC, forced vital capacity.

\section{Statistical Analysis}

All data are expressed as means \pm SD if normally distributed and otherwise as medians with IQRs. Normality of data distribution was checked using the Anderson-Darling and Kolmogorov-Smirnov tests. Student's $t$ test and MannWhitney $U$ test were used to compare variables when appropriate. $p<0.05$ (two-sided) was considered statistically significant. Statistical analysis and figures were created using Prism 9.1.2 (GraphPad Software, San Diego, CA, USA).

\section{Results}

After 4 weeks of add-on therapy with dupilumab, ACT scores had significantly increased from a baseline value of 11.85 \pm 4.58 to $21.30 \pm 3.08$ ( $p<0.0001$, Figure $1 \mathrm{~A}$ ). When compared to the baseline measurement of $58.30 \pm 21.59$, SNOT-22 scores had significantly lowered to $18.90 \pm 16.48$ after 4 weeks $(p<0.0001$, Figure 1B). The better control of respiratory symptoms made it possible to quickly and progressively reduce OCS intake, which was eleminated after 4 weeks of treatment with dupilumab. In fact, within 4 weeks, median prednisone consumption fell from a baseline daily dosage of 12.5 (5-25) $\mathrm{mg} /$ day to $0(0-0) \mathrm{mg} /$ day $(\mathrm{p}<0.0001$, Figure $1 \mathrm{C})$.

In association with these relevant clinical outcomes, we detected parallel improvements in lung function. In particular, prebronchodilator $\mathrm{FEV}_{1}$ had improved from a baseline mean of $2.04 \pm 0.94 \mathrm{~L}$ to $2.30 \pm 0.96 \mathrm{~L}$ after 4 weeks of add-on treatment with dupilumab ( $p<0.01$, Figure $2 \mathrm{~A})$. Furthermore, when compared to baseline, at the week 4 , FVC had significantly risen from $2.85 \pm 1.23 \mathrm{~L}$ to 3.14 $\pm 1.37 \mathrm{~L}(p<0.05$, Figure 2B). Dupilumab also induced a significant reduction in lung hyperinflation caused by airflow limitation. In comparison to baseline, 4 weeks after the first administration, median RV had significantly diminished from $2.78 \pm 0.83 \mathrm{~L}$ to $2.09 \pm 0.75 \mathrm{~L}(p<0.0001$, Figure $2 \mathrm{C})$. RV reduction was associated with a concomitant decrease in TLC, which had declined from $5.93 \pm 1.58$ $\mathrm{L}$ at baseline to $5.47 \pm 1.51 \mathrm{~L}$ after 4 weeks $(p<0.001$, Figure 2D). In addition to such rapid and impressive deflating effects, we also observed that dupilumab had improved small-airway obstruction, with a significant increase in median $\mathrm{FEF}_{25-75}$ from $1.47 \pm 0.85 \mathrm{~L} / \mathrm{sec}$ to $1.80 \pm 0.86 \mathrm{~L} / \mathrm{sec}(p<0.01$, Figure $2 \mathrm{E})$. In the same period, PEF had increased from $5.69 \pm 2.41 \mathrm{~L} / \mathrm{sec}$ to $6.32 \pm 2.25 \mathrm{~L} /$ $\sec (\mathrm{p}<0.01$, Figure $2 \mathrm{~F})$.

With regard to the effects on some biomarkers of type 2 inflammation, in comparison to baseline, median FeNO levels had sharply decreased from $27.50(15.25-33) \mathrm{ppb}$ to 

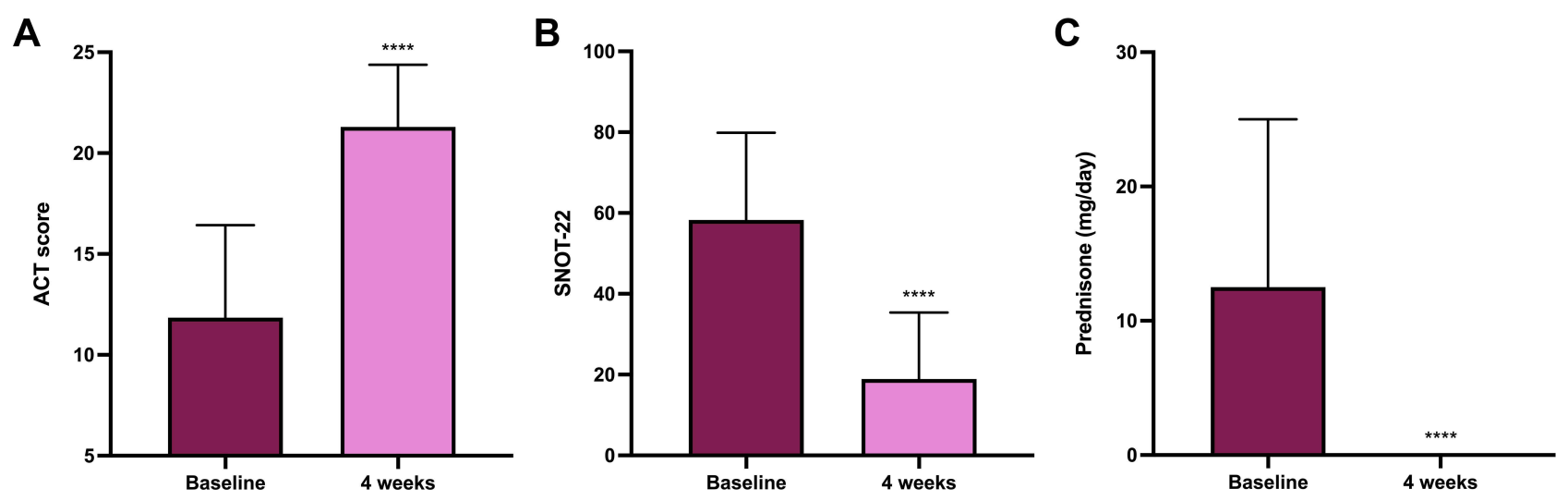

Figure I Clinical effects of dupilumab, with regard to ACT score (A), SNOT-22 score (B), and prednisone intake (C). ACT and SNOT-22 values expressed as means \pm SD; prednisone intake expressed as medians (IQR). $* * * * * p<0.0001$.
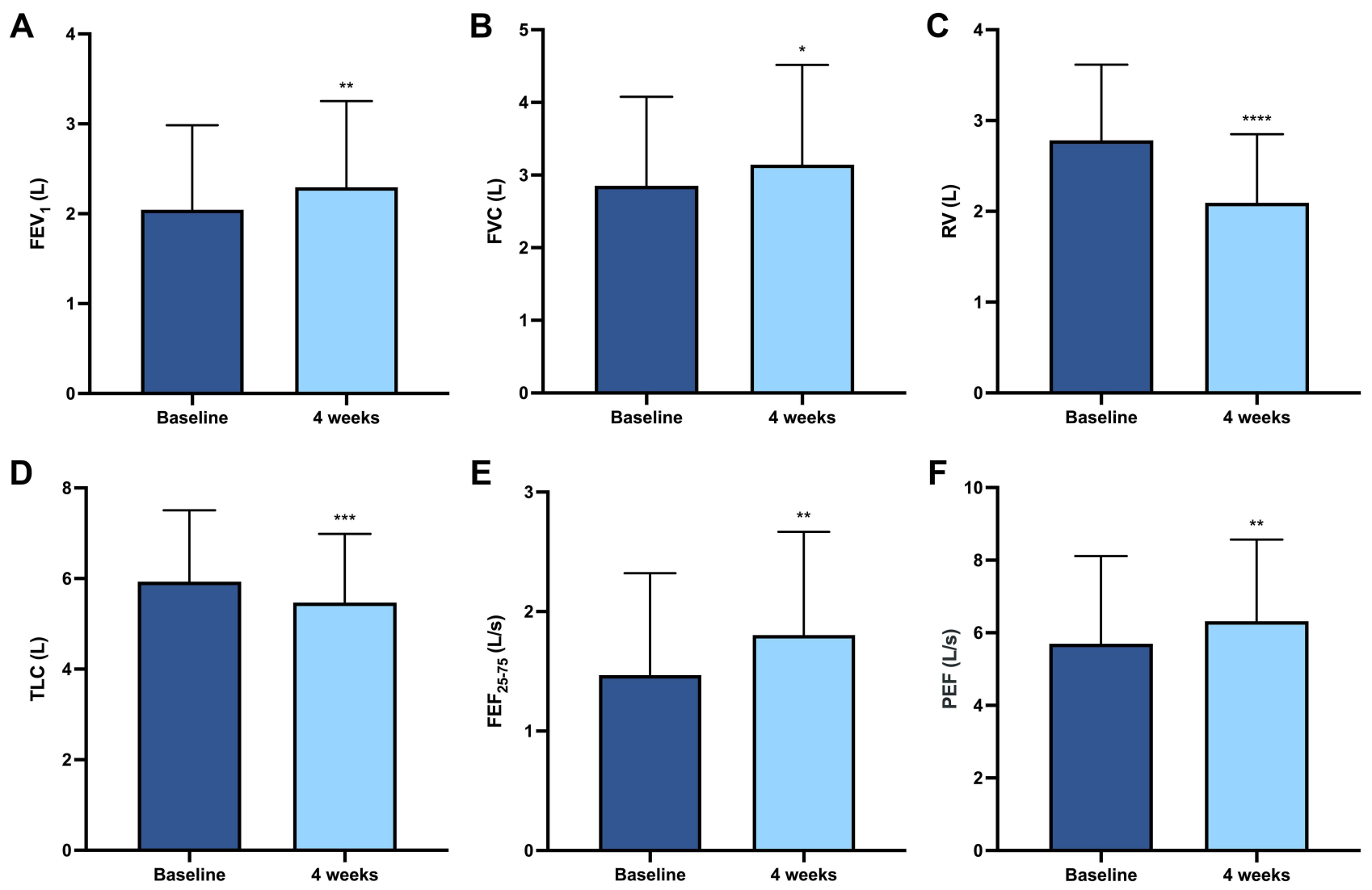

Figure 2 Functional effects of dupilumab with regard to FEV ( $(\mathbf{A}), \mathrm{FVC}(\mathbf{B}), \mathrm{RV}(\mathbf{C}), \mathrm{TLC}(\mathbf{D}), \mathrm{FEF}_{25-75}$ (E), and PEF (F). All parameters expressed as means \pm SD. *p<0.05; $* * p<0.01$; *** $p<0.00$ I; **** $p<0.000$ I.

$2.50(0-6) \mathrm{ppb}(p<0.0001)$ after 4 weeks of treatment with dupilumab (Figure 3A). Moreover, 4 weeks after the first dupilumab administration, median blood eosinophil and basophil counts did not significantly change with respect to baseline, fluctuating from $310(192.5-524.3) \mathrm{cells} / \mu \mathrm{L}$ to $325(215-555)$ cells $/ \mu \mathrm{L}(p=0.81$, Figure $3 \mathrm{~B})$ and from 45 $(30-67.5)$ cells $/ \mu \mathrm{L}$ to $50(30-77.5)$ cells $/ \mu \mathrm{L} \quad(p=0.84$, Figure 3C), respectively.

\section{Side Effects}

Add-on treatment with dupilumab was well tolerated, and no serious adverse reactions occurred throughout this study.

\section{Discussion}

Taken together, the results of this single-center observational study carried out in patients with severe asthma and nasal 

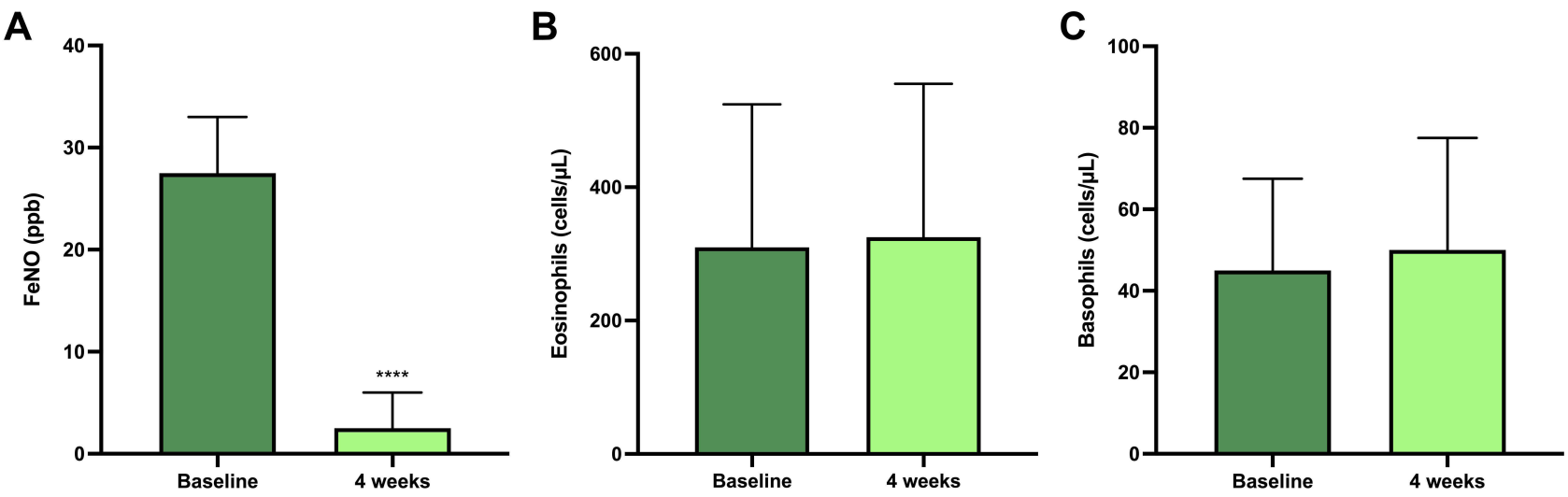

Figure 3 Effects of dupilumab on biomarkers with regard to FeNO (A), blood eosinophils (B), and blood basophils (C). All parameters expressed as medians (IQR). $* * * * p<0.0001$.

polyposis clearly indicate that dupilumab rapidly induced powerful beneficial effects. At 4 weeks after the first administration, this drug had exerted a positive impact on many clinical and functional parameters, as well as on relevant biomarkers of airway type 2 inflammation. Therefore, such findings further corroborate in a real-world setting and in a short time previous data reported by several RCTs and preliminary real-life investigations. ${ }^{25-27,30-32}$

After 4 weeks of treatment, mean ACT score significantly increased in comparison to the low baseline measurement, thus rapidly overcoming the critical threshold of 20 , which reflects adequate asthma control. ${ }^{44}$ This result is consistent with similar data reported by the Liberty Asthma QUEST trial, which showed a very quick improvement in the Asthma Control Questionnaire (ACQ-5) scores already detectable at week 2 of add-on biological therapy with dupilumab. ${ }^{25}$ However, within a real-life context, the ACQ can be usefully substituted by the ACT, which is likely more understandable and easier to be filled in by asthmatic patients routinely referring to clinical centers, who are less strictly monitored than their counterparts enrolled in RCTs. ${ }^{45}$ In fact, the ACT rather than the ACQ has been used in recent real-world studies with the aim of verifying the therapeutic effectiveness of dupilumab in providing satisfactory control of severe asthma symptoms. ${ }^{30-32} \mathrm{We}$ noticed that the marked improvement in asthma control quickly elicited by dupilumab allowed a fast and progressive tapering of prednisone dosage, which within only 4 weeks culminated in a complete withdrawal of OCS treatment, more quickly than that observed in the Liberty Asthma VENTURE study, thereby confirming that reallife findings can be even better than those of RCTs. ${ }^{26,30}$
The suspension of OCS intake is very important for patients with severe asthma, a majority of whom are OCS-dependent and exposed to the negative side effects of OCSs, including adrenal insufficiency, respiratory infections, diabetes, hypertension, osteoporosis, glaucoma, and cataracts. ${ }^{46,47}$ In our patients with severe asthma and CRSwNP, amelioration of clinical symptoms also involved upper airways, as shown by the significant decrease in SNOT-22 scores. This improvement in nasal symptoms confirmed the results of RCTs, thus reiterating the efficacy of dupilumab in the treatment of CRSwNP. ${ }^{27,48}$ We plan to further corroborate and extend these results by evaluating in future studies the effects of dupilumab on a numeric rating scale, endoscopic nasal polyp score, resistance of nasal cavity, and Lund-Mackay computed tomography score.

With regard to lung function, dupilumab rapidly improved airflow limitation in both large and small airways by significantly enhancing prebronchodilator $\mathrm{FEV}_{1}$, FVC, $\mathrm{FEF}_{25-75}$, and PEF. Consistently with both RCT and preliminary real-life observations, ${ }^{25,26,30-32} \mathrm{FEV}_{1}$ increase was remarkable, and after just 4 weeks had neared 300 $\mathrm{mL}$. Interestingly, in our severe asthmatic patients, dupilumab also exerted a fast and effective deflating action, demonstrated by the marked reduction in RV and TLC. This therapeutic effect is very important in severe asthma. Differently from mild-moderate disease, severe asthma is frequently characterized by relevant degrees of lung hyperinflation. ${ }^{49}$

In relation to the effects of dupilumab on biomarkers of type 2 airway inflammation, we found that this monoclonal antibody was able to induce a quick and sharp drop in FeNO levels. Through its peculiar dual-receptor 
antagonism, dupilumab neutralizes the stimulatory actions of IL4 and especially IL13 on iNOS expression at the level of the bronchial epithelium. ${ }^{50,51}$ FeNO is a useful biomarker of type 2 asthma, the levels of which correlate with disease severity, lung-function deterioration, and exacerbation risk. ${ }^{52}$ In addition, FeNO is a reliable tool in choosing tailored biological therapies for severe asthma and monitoring the effects of such treatments. ${ }^{53}$ With regard to observational investigations like ours, FeNO can thus be considered a valuable predictor of therapeutic response to dupilumab detectable in patients with severe asthma, as well as a good biological indicator associated with the clinical and functional outcomes evaluated in these subjects. Differently from FeNO, we did not detect any change induced by dupilumab on blood counts of eosinophils and basophils. These results can be reasonably explained by the distinct biological actions of the various type 2 cytokines. Dupilumab suppresses the functions of IL4 and IL13, which are the main inducers of NO production. Blood and airway eosinophilia are predominantly promoted by IL5, which is not a target of dupilumab. On the other hand, given the small size of our study population, the lack of dupilumab effects on blood eosinophils is not surprising. Indeed, the results reported by two relevant RCTs showed that dupilumab increased blood-eosinophil numbers in relatively low percentages of treated patients, amounting to $4.1 \%$ (versus $0.6 \%$ with placebo) and $14 \%$ (versus 1\% with placebo) in the Liberty Asthma QUEST and Liberty Asthma VENTURE trials, respectively. ${ }^{25,26}$

Another interesting aspect of our present real-life experience is the mixed nature of the study population, which included atopic and nonatopic patients. In both these subgroups, very relevant clinical and functional effects were rapidly elicited by dupilumab, due to its capacity to interfere with allergic and nonallergic pathways underlying type 2 immunomechanisms implicated in airway inflammation. By impeding stimulation of IL4 and IL13 receptors, dupilumab inhibits the bioactivities of the main cellular sources of these cytokines, namely $\mathrm{T}_{\mathrm{h}} 2$ and ILC 2 cells. ${ }^{5}$ ILC 2 and $\mathrm{T}_{\mathrm{h}} 2$ cells are the key players in the integrated cross talk between innate and adaptive immunoresponses underpinning atopic and nonatopic asthma. ${ }^{6,12,54}$ Hence, by dampening such cellular and molecular circuits, dupilumab can extend its therapeutic actions on both upper and lower airways, given the common pathogenic mechanisms shared by type 2 asthma and CRSwNP. ${ }^{54,55}$

In our opinion, the most relevant finding of this reallife study refers to the fast onset of the therapeutic effects of dupilumab. In relation to such a pharmacodynamic pattern, our patients behaved as a quite homogeneous group, highly responsive to the very quick benefits provided by dupilumab. This therapeutic celerity was particularly valuable for those subjects who did not respond satisfactorily to previous biological treatments. It is likely that the early responsiveness experienced by our patients to dupilumab depends on high expression levels of both IL4 and IL13, whose biological actions can be promptly blocked by this monoclonal antibody on several immune/ inflammatory cells. ${ }^{33}$

In conclusion, we herein show in a real-world setting that within 4 weeks of treatment, dupilumab induced in patients with severe asthma and nasal polyposis several therapeutic benefits, including significant improvements in symptom control and lung function associated with a drastic reduction in a viable biomarker of type 2 airway inflammation, such as FeNO. In our opinion, the main strengths of this observational investigation are demonstration of the valuable rapidity of the therapeutic effects prompted by dupilumab with regard to both bronchial and nasal symptoms, assessment of the inhibitory action exerted by dupilumab on lung hyperinflation, detection of similar therapeutic effects in both allergic and nonallergic subjects, and good short-term profile for the safety and tolerability of dupilumab. On the other hand, the most important limitations are quite common to other single-center real-life studies, and include the relatively low number of enrolled patients, as well the lack of randomization design and placebo control.

\section{Authorship Contribution}

All authors contributed to data analysis, drafting, or revising the article, have agreed on the journal to which the article will be submitted, gave final approval to the version to be published, and agree to be accountable for all aspects of the work.

\section{Funding}

This research received no specific grant from any funding agency in the public, commercial, or not-for-profit sectors.

\section{Disclosure}

The authors declare that there are no conflicts of interest in this work.

\section{References}

1. McDowell PJ, Heaney LG. Different endotypes and phenotypes drive the heterogeneity in severe asthma. Allergy. 2020;75:302-310. doi:10.1111/all.13966 
2. Wenzel SE. Severe adult asthmas: integrating clinical features, biology and therapeutics to improve outcomes. Am J Respir Crit Care Med. 2021;203:809-821. doi:10.1164/rccm.202009-3631CI

3. Camiolo MJ, Kale SL, Oriss TB, et al. Immune responses and exacerbations in severe asthma. Curr Opin Immunol. 2021;72:34 42. doi:10.1016/j.coi.2021.03.004

4. Carr TF, Zeki AA, Kraft M. Eosinophilic and noneosinophilic asthma. Am J Respir Crit Care Med. 2018;197:22-37. doi:10.1164/ rccm.201611-2232PP

5. Hammad H, Lambrecht BN. The basic immunology of asthma. Cell. 2021;184:1469-1485. doi:10.1016/j.cell.2021.02.016

6. Matucci A, Bormioli S, Nencini F, et al. The emerging role of type 2 inflammation in asthma. Expert Rev Clin Immunol. 2021;17:63-71. doi:10.1080/1744666X.2020.1860755

7. Tran TN, Zeiger RS, et al. Overlap of atopic, eosinophilic, and TH2high asthma phenotypes in a general population with current asthma. Ann Allergy Asthma Immunol. 2016;116:37-42. doi:10.1016/j. anai.2015.10.027

8. Nelson RK, Bush A, Stokes J, et al. Eosinophilic asthma. J Allergy Clin Immunol Pract. 2020;8:465-473. doi:10.1016/j.jaip.2019.11.024

9. Schleich F, Brusselle G, Louis R, et al. Heterogeneity of phenotypes in severe asthmatics. The Belgian severe asthma registry (BSAR) Respir Med. 2014;108:1723-1732. doi:10.1016/j.rmed.2014.10.007

10. Rossios C, Pavlidis S, Hoda U, et al. Unbiased biomarkers for the prediction of respiratory diseases outcomes (U-BIOPRED) consortia project team. Sputum transcriptomics reveal upregulation of IL-1 receptor family members in patients with severe asthma. J Allergy Clin Immunol. 2018;141:560-570. doi:10.1016/j.jaci.2017.02.045

11. Kuruvilla ME, Lee FE, Lee GB. Understanding asthma phenotypes, endotypes, and mechanisms of disease. Clin Rev Allergy Immunol. 2019;56:219-233. doi:10.1007/s12016-018-8712-1

12. Rodriguez-Rodriguez N, Gogoi M, McKenzie ANJ. Group 2 innate lymphoid cells: team players in regulating asthma. Annu Rev Immunol. 2021;39:167-198. doi:10.1146/annurev-immunol-110119091711

13. Roan F, Obata-Ninomiya K, Ziegler SF. Epithelial cell-derived cytokines: more than just signaling the alarm. $J$ Clin Invest. 2019;129:1441-1451. doi:10.1172/JCI124606

14. Maggi L, Montaini G, Mazzoni A, et al. Human circulating group 2 innate lymphoid cells can express CD154 and promote IgE production. J Allergy Clin Immunol. 2017;139:964-976. doi:10.1016/j. jaci.2016.06.032

15. Froidure A, Mouthuy J, Durham SR, et al. Asthma phenotypes and IgE responses. Eur Respir J. 2016;47:304-319. doi:10.1183/ 13993003.01824-2014

16. Lambrecht BN, Hammad H, Fahy JV. The cytokines of asthma. Immunity. 2019;50:975-991. doi:10.1016/j.immuni.2019.03.018

17. Gour N, Wills-Karp M. IL-4 and IL-13 in allergic airway disease. Cytokine. 2015;75:68-78. doi:10.1016/j.cyto.2015.05.014

18. Ricciardolo FLM, Silkoff PE. Perspectives on exhaled nitric oxide. $J$ Breath Res. 2017;11:047104. doi:10.1088/1752-7163/aa7f0e

19. Pelaia C, Paoletti G, Puggioni F, et al. Interleukin-5 in the pathophysiology of severe asthma. Front Physiol. 2019;10:1514. doi:10.3389/ fphys.2019.01514

20. Pelaia C, Crimi C, Vatrella A, et al. Molecular targets for biological therapies of severe asthma. Front Immunol. 2020;11:603312. doi:10.3389/fimmu.2020.603312

21. Pelaia C, Pelaia G, Crimi C, et al. Biologics in severe asthma. Minerva Med. 2021.

22. Suraya R, Nagano T, Katsurada M, et al. Molecular mechanism of asthma and its novel molecular target therapeutic agent. Respir Investig. 2021;59(3):291-301. doi:10.1016/j.resinv.2020.12.007

23. Pelaia C, Vatrella A, Gallelli L, et al. Dupilumab for the treatment of asthma. Expert Opin Biol Ther. 2017;17:1565-1572. doi:10.1080/ 14712598.2017 .1387245
24. Harb H, Chatila T. Mechanisms of dupilumab. Clin Exp Allergy. 2020;50:5-14. doi:10.1111/cea.13491

25. Castro M, Corren J, Pavord ID, et al. Dupilumab efficacy and safety in moderate-to-severe uncontrolled asthma. $N$ Engl J Med. 2018;378:2486-2496. doi:10.1056/NEJMoa1804092

26. Rabe KF, Nair P, Brusselle G, et al. Efficacy and safety of dupilumab in glucocorticoid-dependent severe asthma. $N$ Engl $J$ Med. 2018;378:2475-2485. doi:10.1056/NEJMoa1804093

27. Bachert C, Han JK, Desrosiers M, et al. Efficacy and safety of dupilumab in patients with severe chronic rhinosinusitis with nasal polyps (LIBERTY NP SINUS-24 and LIBERTY NP SINUS-52): results from two multicentre, randomised, double-blind, placebo-controlled, parallel-group phase 3 trials. Lancet. 2019;394:1638-1650. doi:10.1016/S0140-6736(19)31881-1

28. Kim J, Naclerio R. Therapeutic potential of dupilumab in the treatment of chronic rhinosinusitis with nasal polyps: evidence to date. Ther Clin Risk Manag. 2020;16:31-37. doi:10.2147/TCRM.S210648

29. Kariyawasam HH, James LK, Gane SB. Dupilumab: clinical efficacy of blocking IL-4/IL-13 signalling in chronic rhinosinusitis with nasal polyps. Drug Des Devel Ther. 2020;14:1757-1769. doi:10.2147/ DDDT.S243053

30. Nettis E, Patella V, Lombardo C, et al. Efficacy of dupilumab in atopic comorbidities associated with moderate-to-severe adult atopic dermatitis. Allergy. 2020;10:2653-2661. doi:10.1111/all.14338

31. Dupin C, Belhadi D, Guilleminault L, et al. Effectiveness and safety of dupilumab for the treatment of severe asthma in a real-life French multi-centre adult cohort. Clin Exp Allergy. 2020;50:789-798. doi:10.1111/cea.13614

32. Campisi R, Crimi C, Nolasco S, et al. Real-world experience with dupilumab in severe asthma: one-year data from an Italian named patient program. J Asthma Allergy. 2021;14:575-583. doi:10.2147/ JAA.S312123

33. Bakker DS, van der Wal MM, Heeb LEM, et al. Early and long-term effects of dupilumab treatment on circulating T-cell functions in patients with moderate-to-severe atopic dermatitis. $J$ Invest Dermatol. 2021;141:1943-1953. doi:10.1016/j.jid.2021.01.022

34. Malling HJ. Skin prick testing and the use of histamine references. Allergy. 1984;39:596-601. doi:10.1111/j.1398-9995.1984.tb01979.x

35. Laufer P, Chryssanthopoulos C, Laufer R, Hause LL. The determination of the eosinophil count: comparison of two techniques. J Allergy Clin Immunol. 1987;79:438-441. doi:10.1016/0091-6749(87)90360-5

36. Borzova E, Dahinden CA. The absolute basophil count. Methods Mol Biol. 2014;1192:87-100.

37. Chung KF, Wenzel SE, Brozek JL, et al. International ERS/ATS guidelines on definition, evaluation and treatment of severe asthma. Eur Respir J. 2014;43:343-373. doi:10.1183/09031936.00202013

38. Fokkens WJ, Lund VJ, Mullol J, et al. EPOS 2012: European position paper on rhinosinusitis and nasal polyps 2012. A summary for otorhinolaryngologists. Rhinology. 2012;50:1-12. doi:10.4193/ Rhino50E2

39. Lombardo N, Pelaia C, Ciriolo M, et al. Real-life of benralizumab on allergic chronic rhinosinusitis and nasal polyposis associated with severe asthma. Int $J$ Immunopathol Pharmacol. 2020;34:2058738420950851. doi:10.1177/2058738420950851

40. Wenzel S, Castro M, Corren J, et al. Dupilumab efficacy and safety in adults with uncontrolled persistent asthma despite use of medium-tohigh-dose inhaled corticosteroids plus a long-acting $\beta 2$ agonist: a randomised double-blind placebo-controlled pivotal phase $2 \mathrm{~b}$ doseranging trial. Lancet. 2016;388:31-44. doi:10.1016/S0140-6736(16) 30307-5

41. Graham BL, Steenbruggen I, Miller MR, et al. Standardization of Spirometry 2019 Update. An Official American Thoracic Society and European Respiratory Society Technical Statement. Am J Respir Crit Care Med. 2019;200:e70-e88. doi:10.1164/rccm.201908-1590ST 
42. American Thoracic Society, European Respiratory Society. ATS/ERS recommendations for standardized procedures for the online and offline measurement of exhaled lower respiratory nitric oxide and nasal nitric oxide, 2005. Am J Respir Crit Care Med. 2005;171:912-930. doi:10.1164/rccm.200406-710ST

43. Horváth I, Barnes PJ, Loukides S, et al. A European Respiratory Society technical standard: exhaled biomarkers in lung disease. Eur Respir J. 2017;49:1600965. doi:10.1183/13993003.00965-2016

44. Nathan RA, Sorkness CA, Kosinski M, et al. Development of the asthma control test: a survey for assessing asthma control. J Allergy Clin Immunol. 2004;113:59-65. doi:10.1016/j.jaci.2003.09.008

45. Jia CE, Zhang HP, Lv Y, et al. The asthma control test and the asthma control questionnaire for assessing asthma control: systematic review and meta-analysis. J Allergy Clin Immunol. 2013;131:695-703. doi:10.1016/j.jaci.2012.08.023

46. Heffler E, Blasi F, Latorre M, et al. The severe asthma network in Italy: findings and perspectives. J Allergy Clin Immunol Pract. 2019;7:1462-1468. doi:10.1016/j.jaip.2018.10.016

47. Canonica GW, Colombo GL, Bruno GM, et al. Shadow cost of oral corticosteroids-related adverse events: a pharmacoeconomic evaluation applied to real-life data from the severe asthma network in Italy (SANI) registry. World Allergy Organ J. 2019;12:100007. doi:10.1016/j.waojou.2018.12.001

48. Bachert C, Hellings PW, Mullol J, et al. Dupilumab improves healthrelated quality of life in patients with chronic rhinosinusitis with nasal polyposis. Allergy. 2020;75:148-157. doi:10.1111/all.13984
49. Jarjour NN, Erzurum SC, Bleecker ER, et al. Severe asthma - lessons learned from the National Heart, Lung and Blood Institute severe asthma research program. Am $J$ Respir Crit Care Med. 2012;185:356-362. doi:10.1164/rccm.201107-1317PP

50. Carr TF, Kraft M. Use of biomarkers to identify phenotypes and endotypes of severe asthma. Ann Allergy Asthma Immunol. 2018;121:414-420. doi:10.1016/j.anai.2018.07.029

51. Busse WW, Kraft M, Rabe KF, et al. Understanding the key issues in the treatment of uncontrolled persistent asthma with type 2 inflammation. Eur Respir J. 2021;4:2003393. doi:10.1183/13993003.033 93-2020

52. Ulrik CS, Lange P, Hilberg O. Fractional exhaled nitric oxide as a determinant for the clinical course of asthma: a systematic review. Eur Clin Respir J. 2021;8:1891725. doi:10.1080/20018525.2021.1891725

53. Rolla G, Heffler E, Pizzimenti S. An emerging role for exhaled nitric oxide in guiding biological treatment in severe asthma. Curr Med Chem. 2020;27:7159-7167. doi:10.2174/0929867327666200 713184659

54. Scadding GK, Scadding GW. Innate and adaptive immunity: ILC2 and Th2 cells in upper and lower airway allergic diseases. J Allergy Clin Immunol Pract. 2021;9:1851-1857. doi:10.1016/j. jaip.2021.02.013

55. Matucci A, Bormioli S, Nencini F, et al. Asthma and chronic rhinosinusitis: how similar are they in pathogenesis and treatment responses? Int J Mol Sci. 2021;22:3340. doi:10.3390/ijms22073340z

\section{Publish your work in this journal}

The Journal of Asthma and Allergy is an international, peer-reviewed open-access journal publishing original research, reports, editorials and commentaries on the following topics: Asthma; Pulmonary physiology; Asthma related clinical health; Clinical immunology and the immunological basis of disease; Pharmacological interventions and new therapies. The manuscript management system is completely online and includes a very quick and fair peer-review system, which is all easy to use. Visit http://www.dovepress.com/testimonials.php to read real quotes from published authors. 